\title{
Reduced Laryngeal Sensitivity and its Association with Dysphagia and Cough in Children with Neurological Impairment
}

\author{
Nadine Freitag ${ }^{1}$, Pia Tews ${ }^{1}$, Nicole Hübl ${ }^{1}$, Katrin Krug $^{2}$, Julia Kristin ${ }^{1}$, Felix Distelmaier ${ }^{1}$, \\ and Dirk Schramm ${ }^{1}$ \\ ${ }^{1}$ Medical Faculty, University Hospital Düsseldorf \\ ${ }^{2}$ RWTH Aachen University Medical Faculty
}

June 24, 2021

\begin{abstract}
Background Silent aspirations are frequent in children with neurological impairment. They dramatically increase the risk for acute and chronic respiratory insufficiencies leading to high morbidity and mortality. Laryngeal sensitivity deficits have been linked to aspirations in adults and are a suspected cause for dysphagia in children. In a similar neurological circuit as swallowing, laryngeal receptors trigger coughing as a protective airway reflex. The aim of this study was to examine the association between reduced laryngeal sensitivity, aspiration and coughing in neurologically impaired children. Design and Methods In a retrospective study, 110 children with suspected dysphagia who received a clinical evaluation of swallowing and a flexible endoscopic evaluation of swallowing (FEES) between 2013 and 2019 in the children's university clinic Düsseldorf were analyzed. Laryngeal sensitivity was tested by the endoscopic touch method. Fifty-four patients (49.1\%) had neurological impairments, 56 patients $(5.9 \%)$ had no or other comorbidities and served as a control cohort. Associations were computed using $\times 2$-test. Results Children with neurological impairment suffered from laryngeal sensory deficit significantly more often and seemed to cough less frequently than children with no or other comorbidities. Reduced laryngeal sensitivity could not be correlated to less coughing. Coughing acted as a predictor of aspiration only in the neurologically impaired group of children with reduced laryngeal sensitivity. Conclusion Reduced laryngeal sensitivity is a potential cause of silent aspirations in children with neurological impairment. However, reduced laryngeal sensitivity did not lead to significantly less coughing which might be due to a lack of discrimination between different levels of sensitivity deficits by the endoscopic touch.
\end{abstract}

\section{Hosted file}

Original research_Freitag et al_Reduced Laryngeal Sensitivity and its Association with Dysphagia and Co available at https://authorea.com/users/421516/articles/527521-reduced-laryngealsensitivity-and-its-association-with-dysphagia-and-cough-in-children-with-neurologicalimpairment

\section{Hosted file}

Tables_Freitag et al_Reduced Laryngeal Sensitivity and its Association with Dysphagia and Cough in Chil available at https://authorea.com/users/421516/articles/527521-reduced-laryngealsensitivity-and-its-association-with-dysphagia-and-cough-in-children-with-neurologicalimpairment 